142

The
Comedical
Cournal

RESEARCH ARTICLE

\title{
Transportation of Donated Hearts by Drone in Comparison to Road-Bound Vehicles in Mexico City
}

\author{
Muhammad Al-Ayyad ${ }^{1}$, Amani Al-Ghraibah",* and Husam Alkhatib ${ }^{2}$ \\ ${ }^{I}$ Medical Engineering Department, Al-Ahliyya Amman University, Amman, Jordan \\ ${ }^{2}$ Medical Engineering Department. KTH Royal Institute of Technology, Stockholm, Sweden
}

\begin{abstract}
:
Background:

Organ transportation between hospitals has many challenges since the harvested organ needs to be transplanted to the recipient within an optimal time frame. It is important to transfer the organ from the donor to the recipient site quickly and professionally.

Aim:

The aim of this research is to investigate whether the transportation of donated hearts by drones is more efficient than transportation using roadbound vehicles.

Methods:

A simulation using MatLab software was performed to calculate the heart transportation time between three different hospitals in Mexico City by road-bound vehicles and by drones. This simulation was performed to demonstrate how congestion and traffic in Mexico City prevent healthy hearts from being transplanted to patients on time. The 24-hour period was divided into four 5-hour periods and these were chosen to be: Period 1 (22:00-03:00), Period 2 (04:00-09:00), Period 3 (10:00-15:00) and Period 4 (16:00-21:00).

Results:

The time it took to travel between the hospitals by car within each of the 5-hour periods was calculated. It was observed that, in period 2 , a high volume of traffic was present causing road-bound vehicles to take a longer time to deliver hearts to hospitals. All hospitals were within reach of each other if the hearts were to be transported by drone and the delivery time by drone in all cases was less than 1.88 hours, which is the optimum time for the harvested heart to be transported without risk from the donor to the recipient.

\section{Conclusion:}

Using drones will increase the safety of transplants, reduce the heart transplantation waiting list and eventually, heart transplantation may be possible in different areas of the city at rush hour times.
\end{abstract}

Keywords: Drone, Organ transportation, Heart transplantation, Harvested heart delivery, Road-bound vehicles, Lengthen.

\begin{tabular}{|l|l|l|l}
\hline Article History & Received: August 13, 2019 & Revised: October 14, 2019 & Accepted: October 17, 2019
\end{tabular}

\section{INTRODUCTION}

Heart transplantation is the gold standard in the treatment of patients with end-stage heart failure. Even though there have been great advances in technology within the cardiac field, treatment options such as mechanical devices or medical therapy have not been able to provide long-term results which are as good as heart transplantation. Heart transplantation is, however,

* Address correspondence to this author at the Medical Engineering Department, Al-Ahliyya Amman University, Amman, Jordan;

E-mail: a.ghraibah@ammanu.edu.jo only an option for a limited number of patients since the number of patients with severely advanced heart failure is increasing more rapidly than the number of available donor hearts [1]. This imbalance between supply and demand is causing organ waiting lists to lengthen.

In organ transplantation, it is important that the organ should be received by a patient who is the best possible match. Many different medical and logistical characteristics must be considered. The general matching criteria for all types of organ transplantation are: matching blood type, matching organ size, 
time spent waiting for transplantation and the relative distance between donor and recipient. For heart transplantation, there are also specific criteria including the medical urgency for the recipient, the degree of immune system match between donor and recipient, and whether the recipient is a child or an adult [2]. Organs are first distributed locally, and if no match is found within the local area, the organs are then offered regionally and then nationally until a good organ recipient match is found.

Ischemia time is the time period when the donated organ is not supplied with oxygen or nutrients, which will lead to heart damage [3]. For a heart, an ischemia time beyond 4 hours is associated with reduced patient survival, therefore a harvested heart should be transplanted into the recipient within 4 hours. In a study examining six cases, it was shown that the mean period between clamping, which initiates the ischemic period for the heart, and harvesting of the heart ranged between 20 and 40 minutes [4]. In a study where a total of 1491 heart transplants were analyzed, it was shown that the median surgical implant time ranged from 71 minutes to 87 minutes [3]. Rapid allocations of organs and procurement techniques could reduce ischemia times [4].

When a heart becomes available at one hospital, the medical teams must quickly transport the heart to the hospital where the recipient is located [5]. Transportation of organs is mainly carried out by road-bound vehicles [6]. In large cities such as megacities, with a population of at least 10 million inhabitants [7], the high number of cars on the roads will cause traffic issues and congestion that increase the transportation time of the organ from donor to recipient hospital. To lessen this problem in India, "green corridors" have been implemented; police and other authorities allow the organ transporting vehicles to pass through traffic quickly by keeping the traffic lights green and keeping cars out of the way. As the volume of transplants increases in India, this will not be a lasting solution $[5]$.

If organs need to be transported over longer distances or geographical features prohibit rapid transportation via roads, airborne vehicles can be used. The use of airborne vehicles for organ transportation is a complex operation and a great deal of logistical planning is required. Many stakeholders and multimode vehicles are involved when two or more transportation methods are used. Often airborne vehicles have to be available at short notice and many people are involved in the transportation of the organ. It has been shown that multimodal transportation increases the risk and the level of uncertainty because there are many different people and vehicles involved in the transportation chain [6]. Mode of transportation must be chosen carefully when transporting an organ since the ischemia time, which is dependent on transportation time, affects the success rate of heart transplan-tation. A problem for logistical planning is that it is not easy to predict exactly when a donor will die. Therefore, it is hard to know when the organ will be available for donation [8]. Organizational factors such as transportation arrangements and organ allocation can affect ischemia time [3] and therefore patient survival.

Recently, Unmanned Aerial Vehicles (UAVs) have been used in the healthcare transportation system for delivery of medical supplies to disaster areas where access is restricted, and to safely deliver disease test samples in areas of contagion [9]. Delivery drones carrying defibrillators have also been used to save the lives of people suffering from cardiac arrest. Drones with defibrillators have the ability to arrive more quickly than road-bound emergency vehicles, increasing the chances of patient survival [10]. UAvs may be beneficial for transportation of goods in a rapid, safe and cost-efficient manner within the healthcare sector [9]. Currently, several trials are ongoing to build drones specialized in organ delivery, therefore in the near future, this dream will come true.

\subsection{Transplantation in Mexico City}

Mexico has low transplant rates compared to other countries in Latin America; this may be due to a complex health system divided into subsystems. Each subsystem covers different sectors of the population, for example, there is a healthcare subsystem for employees of the state and another one for employees in the private sector. Organs from a donor in one subsystem are usually only provided for recipients in that same subsystem. In most cases, there is no communication among the health subsystems with regard to transplantation. Transplant possibilities are therefore limited by the number of donor and recipient matches that are available within each subsystem. At present, all organ donors must be transferred to an organ transplant licensed hospital. This complicates the process of organ donation since most hospitals are not licensed [11].

Three of the largest hospitals, which not only are licensed to perform heart transplantations in Mexico, but also perform a substantial amount of heart surgery are La Raza, ABC Medical Center and the General Hospital of Pachuca. La Raza and ABC Medical Center are located within Mexico City, and the General Hospital of Pachuca is located in an adjacent city called Pachuca. The General Hospital of Pachuca is the largest of these three and is funded by the state. Likewise, La Raza hospital is funded by the state; however, this hospital is renowned for heart transplantations and has a strong reputation for success within this field [12]. ABC Medical Center, on the other hand, is privately funded; moreover, this hospital is a leader in medical technology and patient service in Mexico City [13]. At the time of writing this paper, the waiting list for heart transplantation in Mexico comprised 47 patients. In 2017, a total of 33 heart transplants were performed in Mexico (until 18 December 2017) [14, 15].

A recent study in rush hour traffic congestion concluded that globally, Mexico City is the second worst city for rush hour traffic after Bangkok, Thailand. Likewise, it has been approximated that, in the chaotic Aztec capital, a journey during the rush hour may take more than 58 minutes, instead of an average 30 minutes travel time respecting the speed limit $[16,17]$. Accidents and other disruptions can further affect the travel time between relatively close locations. Therefore, it is challenging to accurately calculate the travel time of roadbound vehicles. Even though Google Maps and other route planners take into account factors such as traffic and weather conditions at that particular time of day when calculating route times, for places such as Mexico City, it is extremely difficult 
to do so with high precision.

\subsection{Heart Transplantation}

Heart transplantation is a race against time since a harvested heart needs to be transplanted to the recipient within an optimal time frame of 4 hours. When a heart becomes available at a particular hospital, the medical team must move quickly to transport the heart to the hospital where the recipient is located [5]. The two main transportation methods for organs are by road or air. Road-bound vehicles are more commonly used, but when an organ needs to be transported over longer distances or where geographical features prohibit rapid transportation via roads, airborne vehicles are used. The use of current airborne vehicles to transport organs is a complex operation that includes much coordination and planning. It often requires multimode transportation and an aircraft must be available at short notice [6]. The demand for organs is large; every 10 minutes, someone is added to the national transplant waiting list in the USA and, on average, 20 people die each day in the USA while waiting for transplants [18]. One of the greatest challenges within organ transplantation is moving the organ from the donor to the recipient efficiently [5]. At present, 8 out of 10 available donated hearts do not make it to the recipient due to a variety of factors, the most common being the distance between hospitals resulting in prolonged transportation time [19]. To grant transplant recipients the healthiest outcome, it is of extreme importance that the highest level of service and efficiency be guaranteed [6].

The aim of this project is to investigate whether the transportation of hearts by drones is a more efficient method than the transportation of hearts by road-bound vehicles. As an airborne transportation method, drones could improve logistical planning in the supply chain management of harvested heart delivery. To study this possibility, simulations have been performed using MatLab (MathWorks, Natick, MA, USA). This project simulates the transportation of hearts between three different hospitals in Mexico City by road-bound vehicles and by drones.

\section{METHODOLOGY}

To gain an understanding of the logistics of heart transplantation and to obtain accurate data to use for the simulation, published scientific papers and web pages have been reviewed. System identification of heart transplantations was carried out by drawing flow diagrams based on knowledge gained with regard to the subject. Transportation of donated hearts was part of the system that was chosen to investigate further. The level of abstraction was chosen to be the transportation of donated hearts. A simulation model was therefore created in MatLab. This simulation was performed to investigate and demonstrate how congestion and traffic in Mexico City prevent healthy hearts from being transplanted to patients in need. Thus, the simulation aimed to prove that all hearts could be successfully transplanted if all transportation of donated hearts was carried out by drone instead of road-bound vehicles. The level of detail about the heart was chosen to be none for simplification purposes and it was assumed that all hearts from donors were successfully implanted in the recipients.
Hence, the first step performed was to study and analyze the congestion and traffic times between the three selected hospitals. Thus, transportation times were studied. To investigate how the transportation time by drone and car varied over 1 day, a 24-hour period was divided into four 5-hour periods: Period 1 (22:00-03:00), Period 2 (04:00-09:00), Period 3 (10:00-15:00) and Period 4 (16:00-21:00). The time it took to travel by car between the hospitals within each 5 -hour period was calculated. The calculation was done by taking the time predicted by Google Maps [20] for car travel between the hospitals for each hour within the period and taking an average of all 5 hours. The data in this work was extracted from Google Maps namely the website link: https://www.google.se /maps/preview. The time taken for a drone to travel between each hospital was calculated by measuring the distance between the hospitals using an online tool called FreeMapTools [21, 22]. The traveling time was calculated for a particular type of UAV, the "Hercules Heavy Lift" UAV, which has average traveling speed of $51.499 \mathrm{~km} / \mathrm{h}$ [23].

The aim of this study was to demonstrate how heavy road traffic could lead to a donor heart being no longer transplantable, so it was decided to use the period in which this could be clearly seen. As a result, Period 2 (04:00-09:00) was chosen to be used in the simulation as it was observed that traffic congestion was high in this period and caused roadbound vehicles to take longer times to deliver hearts to target hospitals.

Three hospitals were chosen due to their size and reputation for heart transplantation in Mexico - the General Hospital of Pachuca, La Raza, and ABC Medical Center. For simulation purposes, it was assumed that all were capable of performing heart organ transplantations in Period 2. In addition, it was assumed that, apart from transporting hearts between one another, the three hospitals could also deliver hearts internally. In this situation, for simplification, it was assumed that transportation required no time.

Once the above parameters had been set, the simulation was programmed. The first part programmed in this simulation was to make each hospital capable of generating a supply of hearts, which represented the number of hearts that came from the number of deceased heart donors at that specific hospital in that particular period. The supply at each hospital was generated with a MatLab random function, randomizing numbers from zero to a particular maximum number of hearts, which was dependent on the size of that hospital. It was assumed that, in that particular period, the General Hospital of Pachuga could supply up to six hearts due to its size, while La Raza hospital and $\mathrm{ABC}$ Medical Center, could only supply up to two and three hearts, respectively, as they were smaller hospitals.

Each hospital was then also given a demand, which represented the number of people in need of a heart at that particular hospital, in that particular period. This was also generated with a MatLab random function randomizing numbers from zero to a particular maximum number of hearts. It was assumed that, in that particular period, the General Hospital of Pachuca could demand from zero to four hearts, La Raza could demand between zero and eight, and $\mathrm{ABC}$ Medical Center between zero and three. La Raza was given a higher 
demand than the other two hospitals because it was a very highly specialized and renowned hospital for heart transplantation. Therefore, the number of people waiting for a heart would be higher. On the other hand, it was assumed that due to the size of the General Hospital of Pachuca and because it is a state-funded hospital, there would be a larger supply of hearts in comparison to the demand (need). Hence, the values of supply and demand were chosen relevant to the background study performed on each hospital (see Introduction section).

From the study and analysis of the heart transplantation operational chain from the literature review, the time for which a heart can be transported, without increased risk to patient survival, was determined and calculated to be 1 hour 53 minutes ( 1.88 hours). This maximum transportation time was calculated by subtracting the maximal clamping to harvest time and the maximal surgical time from the maximal ischemia time that is not associated with reduced patient survival. Thus, the transportation time allowed was calculated by Eq. (1):

Allowed transportation time $=($ maximal clamping to harvest time +

maximal surgical time) - maximal ischemia time

The simulation used the calculated transportation time to check whether the time between hospitals complied with this limiting transportation time and thus verified which hospitals were within reach of each other, i.e. if the time between two hospitals was greater than 1.88 hours, hearts could not be transported between those two hospitals. Once this verification was made, then the random supply and demand of hearts generated in that particular period for each hospital were compared. Hence, the simulation was then able to recognize whether a particular hospital had an extra supply of hearts that could be donated to a hospital in need of extra hearts, of course within the 1.88 hour limit.

Therefore, the simulation was programmed with the aim that it could generate different outcomes depending on the different combinations possible in respect of the supply and demand of each hospital. In other words, the simulation was programmed to recognize if a hospital could donate hearts to another hospital depending on the different combinations of supply and demand generated at each hospital and the time limit of 1.88 hours. The simulation could, therefore, recognize many different combinations of supply and demand and provide different outcomes.

In order to create a comparative and visual result, a graph was then plotted. The graph was based on output data from the simulation and was created in Microsoft Excel. The graph plotted the service level for road-bound vehicles and for drones for each run, in which a run represented 1 day. The service level was calculated to represent a percentage of the total number of transplanted hearts in that period out of the potential total number of hearts transplantable in that period. Thus, the service level was calculated by Eq. (2):

$$
\text { Service level }=\frac{\text { Total no. of transplanted hearts in that period }}{\text { Total no. of hearts available in that period }}
$$

The simulation was run 31 times, simulating a full calendar month. For simplification purposes, when writing the code, the Hospital General of Pachuca was given the Letter A, La Raza Hospital was given the Letter B, and ABC Medical Center was given the Letter $\mathrm{C}$.

\section{RESULTS}

\subsection{Transportation Times}

The following figures show the differences in transportation times between different combinations of two hospitals using drones or road-bound vehicles. The transportation or "delivery time" as shown on the graphs is given in hours on the $y$-axis. The time of day, which is divided into the four periods, is represented on the $x$-axis. The blue columns represent "Road-bound vehicle transportation" while the orange columns represent "Drone transportation". Figs. (1 and 2) show the results of transporting the organs from the General Hospital of Pachuca to La Raza Hospital and back, in the four different periods of time during the day. The figures show that the drone had a very similar traveling time to roadbound vehicles in almost all cases seen in Figs. (1 and $\mathbf{2}$ ). Also, in all periods of the day, the drone took the same time traveling from one point to another and back, which was about 1.6 hours.

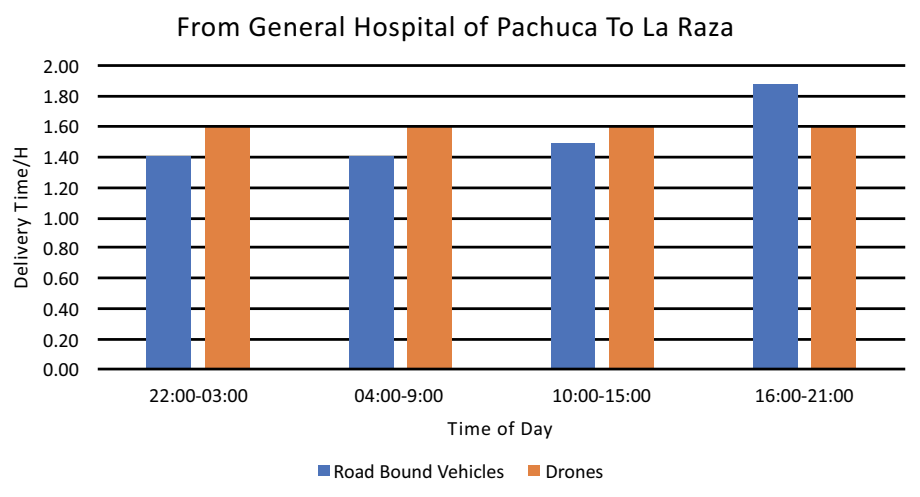

Fig. (1). Traveling times between the General Hospital of Pachuca and La Raza Hospital. 


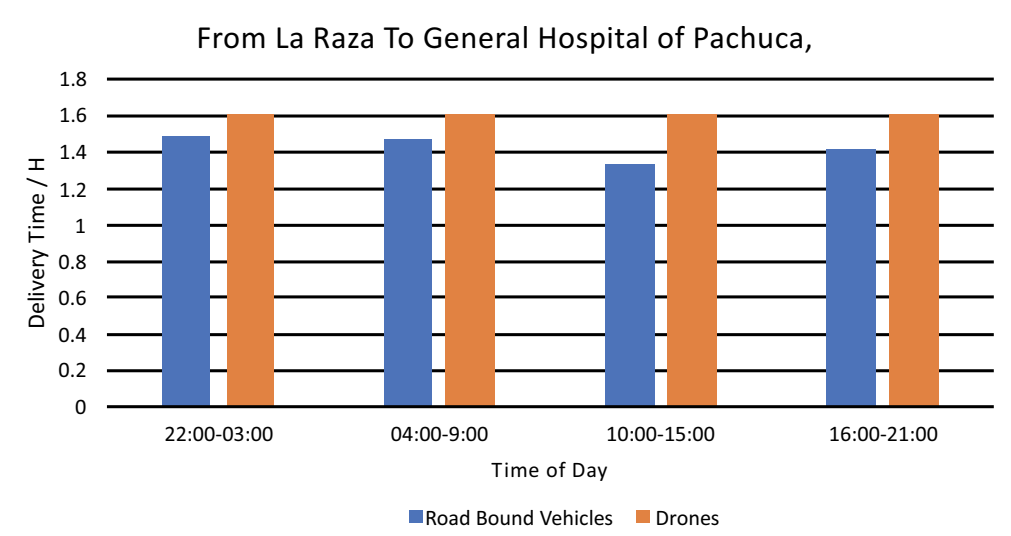

Fig. (2). Traveling times between La Raza Hospital and the General Hospital of Pachuca.

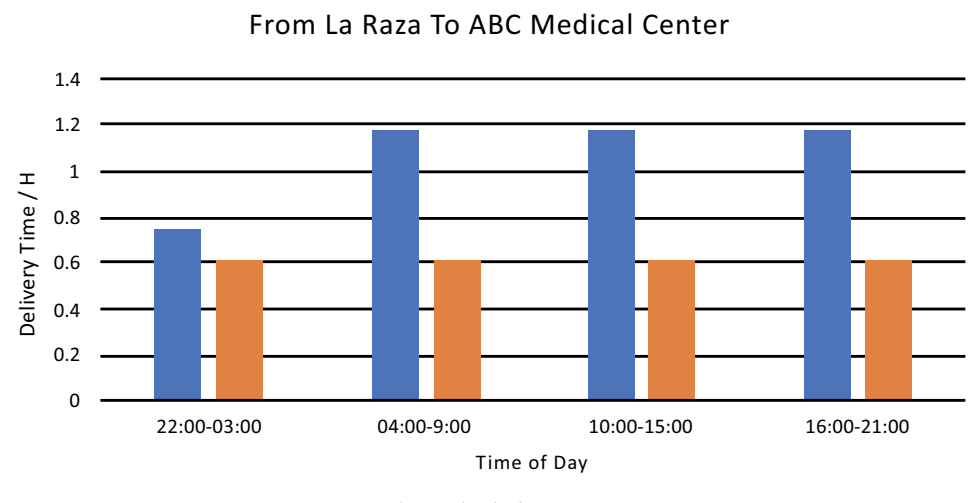

noad Bound Vehicles $\quad$ Drones

Fig. (3). Traveling times from La Raza to ABC Medical Center.

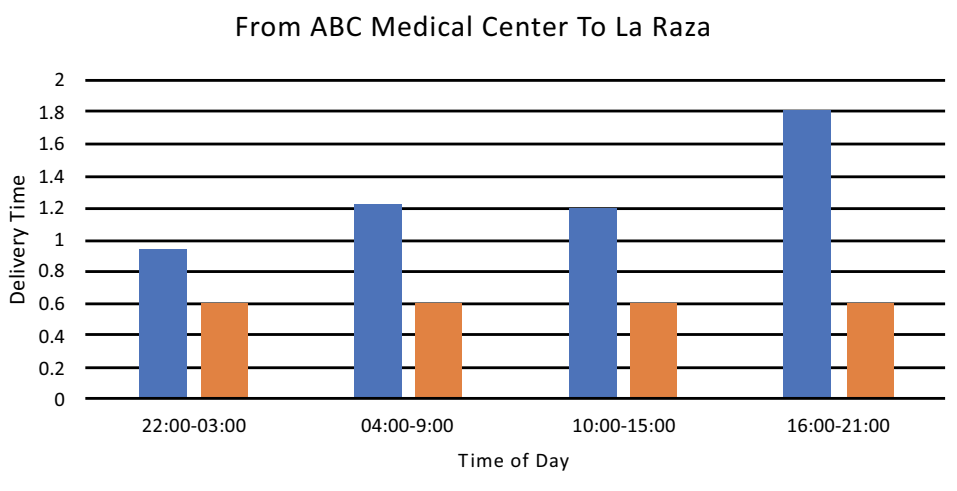

- Road Bound Vehicles Drones

Fig. (4). Traveling times from $A B C$ Medical Center to La Raza.

Figs. (3 and 4) show the traveling times for the donated organs from La Raza Hospital to ABC Medical Center and back using both road-bound vehicles and drones. In all periods during the day, the drone took less time than road-bound vehicles and it was around 0.6 hours as seen in both figures.

Figs. (5 and 6) show the traveling times for the organs from ABC Medical Center to the General Hospital of Pachuca and back. As in Figs. (3 and 4), the results here showed that the drone spent less time traveling than the road-bound vehicles, approximately 1.8 hours in all time periods.

From the previous figures, it can be seen that, during Period 2 (from 04:00 to 09:00), all hospitals were within reach of each other for the exchange of hearts if the hearts were transported by drones since the delivery time by drone was always less than 1.88 hours; however, between some hospitals (the General Hospital of Pachuca and ABC Medical Center), the transportation times by road-bound vehicles exceeded 1.88 hours. 


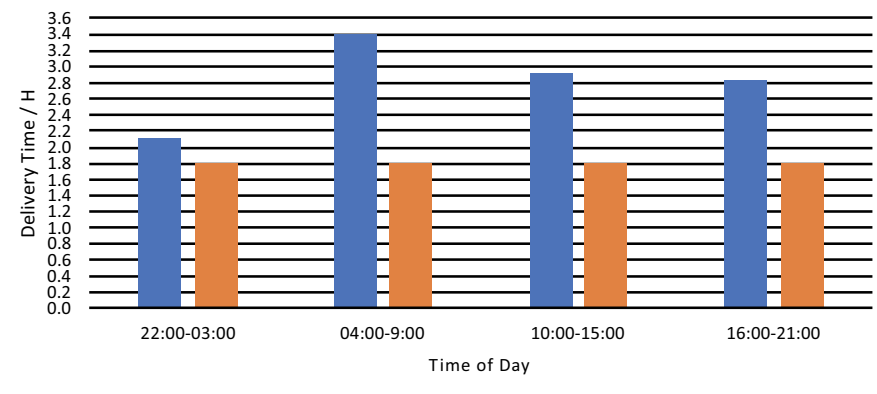

ERoad Bound Vehicles Erones

Fig. (5). Traveling times between the General Hospital of Pachuca and ABC Medical Center.

From ABC Medical Center To General Hospital of Pachuca,

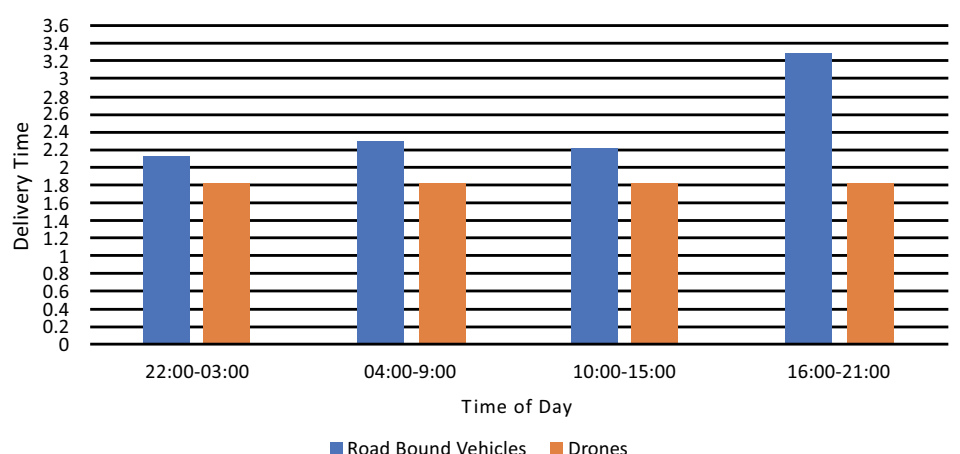

Fig. (6). Traveling times from $A B C$ Medical Center to the General Hospital of Pachuca.

\section{2.. Simulation Example - 08:00 within Period 2}

The results from the simulation varied depending upon the time of day selected. An example result is given in Fig. (7) when the simulation was run for 08:00 within Period 2. As seen in Fig. (7), the simulation first demonstrated which hospitals could be reached by each respective hospital at 08:00. Then the simulation generated a random number of hearts supplied at each respective hospital, as seen in Fig. (7). Thus, A (the General Hospital of Pachuca) could supply one heart, B (La Raza) had no hearts to supply and C (ABC Medical Center) could supply three hearts. Likewise, the simulation generated a random number of demands for hearts at each particular hospital. In this case, A was in need of one heart, B was also in need of one heart, and $\mathrm{C}$ was in need of four hearts.

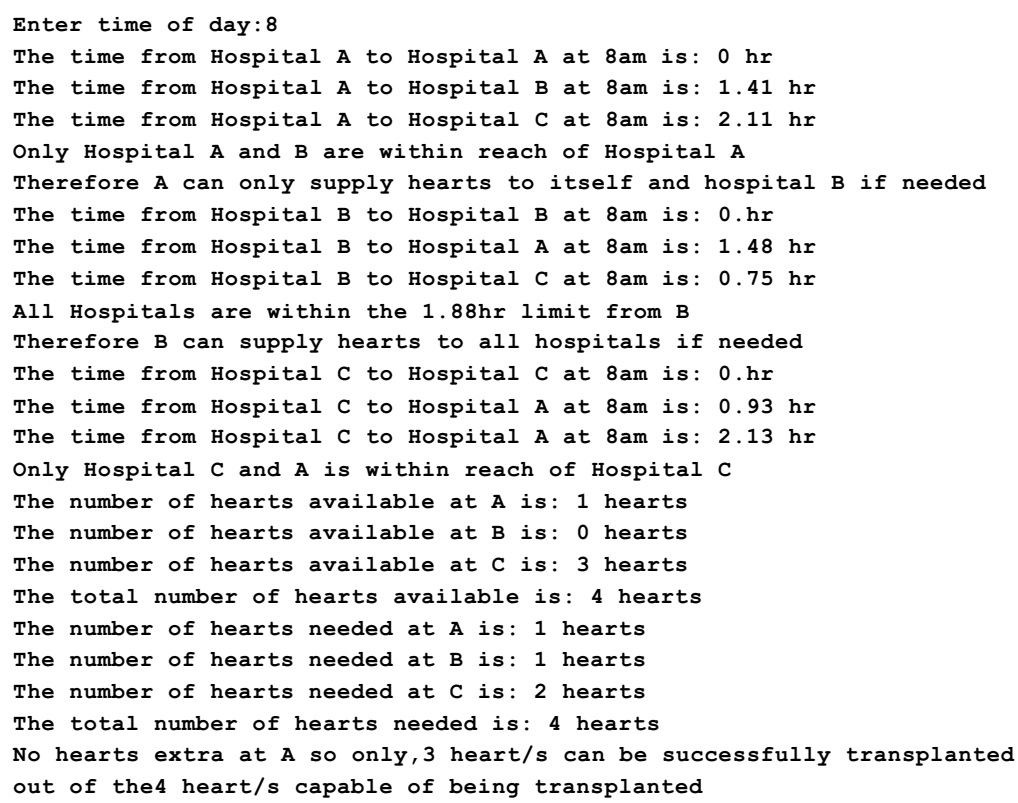

Fig. (7). The command window from MatLab presents the outcome of simulation for 1 day at 08:00 (Period 2). 
Therefore, A (the General Hospital of Pachuca) could meet its own demand with its own supply as it had one heart available and it was in need of one heart. Thus, no extra hearts were available at this hospital, yet one successful heart transplant could take place here. On the other hand, Hospital B (La Raza) was not capable of meeting its own demand with its own supply, as it had no hearts available. However, in this simulation, at that particular time, La Raza (B) had one recipient patient in a need of a heart. In this case for Hospital $\mathrm{C}$ (ABC Medical Center), three hearts were available and were ready to be transplanted.

In terms of demand, in this particular case, the $\mathrm{ABC}$ Medical Center had two patients in need of hearts, meaning that two hearts could be delivered to their own cardiac ward for two successful transplants. Thus, in turn, the simulation recognizes that $\mathrm{C}$ ( $\mathrm{ABC}$ Medical Center), had one heart that could be sent to a reachable hospital. The ABC Medical Center could, therefore, send its extra supply of transplantable hearts to B (La Raza Hospital). However, because as noted by the simulation Fig. (7), B is not within the 1.88 hour limit from C, therefore this extra heart could not be delivered to B by a roadbound vehicle.

Hence, the simulations observed and calculated how many hearts could be successfully transplanted using road-bound vehicles. Therefore, as seen in Fig. (7), only three hearts could be successfully transplanted out of the four potentially transplantable hearts. Thus, the service level for this particular case was $3 / 4(75 \%)$ and one patient had to be kept on the waiting list for a transplantable heart.

On the other hand, if the transportation was made by drone instead of a road-bound vehicle, all hospitals would have been within reach of one another. Hence, by drone, the ABC Medical Center could have delivered its extra heart to La Raza Hospital and thus, the service level for that particular time of day would be $4 / 4(100 \%)$, as all transplantable hearts could be successfully delivered where needed.

Each of the 31 runs produced figures for supply, demand and successful transplants. The 31 runs of the simulation generated data to produce a graph that shows the service levels for all three hospitals combined for each run Fig. (8).

\section{DISCUSSION}

Time period 2 (04:00 am to 09:00 am) was chosen for the simulation as data from this period showed the heaviest traffic in Mexico City. Thus, between these hours, transportation by road-bound vehicles is extremely slow. Transportation time by drones is not affected by heavy traffic so the time period chosen clearly shows the benefit of using drones during heavy traffic hours. However, the impact on transporting donated hearts by a drone during other time periods of the day has not been investigated. It can be assumed that the transportation of donated hearts with drones will be most common during the time of day when heavy traffic is present. Moreover, in some time periods when there is no traffic, an ambulance can deliver organs within the optimal time and there is actually no need to transport hearts by drone, as seen in Fig. (8).

Additionally, the level of abstraction used in this simulation only represents a small part of the whole system that applies during heart transplantation. There are many parts of the supply chain that are excluded in the simulation. Parts of the supply chain that are not included, such as handling and testing of the heart, can affect the overall time that the heart is out of a body and would, therefore, affect the result of this simulation.

The information within the level of abstraction used was limited in this simulation. One assumption that was made is that all hearts that are supplied have eligible recipients and that the transplantation was successful. Unfortunately, this is not always the case in reality. No matter how drones could impact the delivery of hearts between hospitals during a period of heavy traffic, a clinical result must not be assumed. However, if clinical parameters, such as the possibility of donor-recipient match, were taken into account, the simulation would more accurately depict reality.

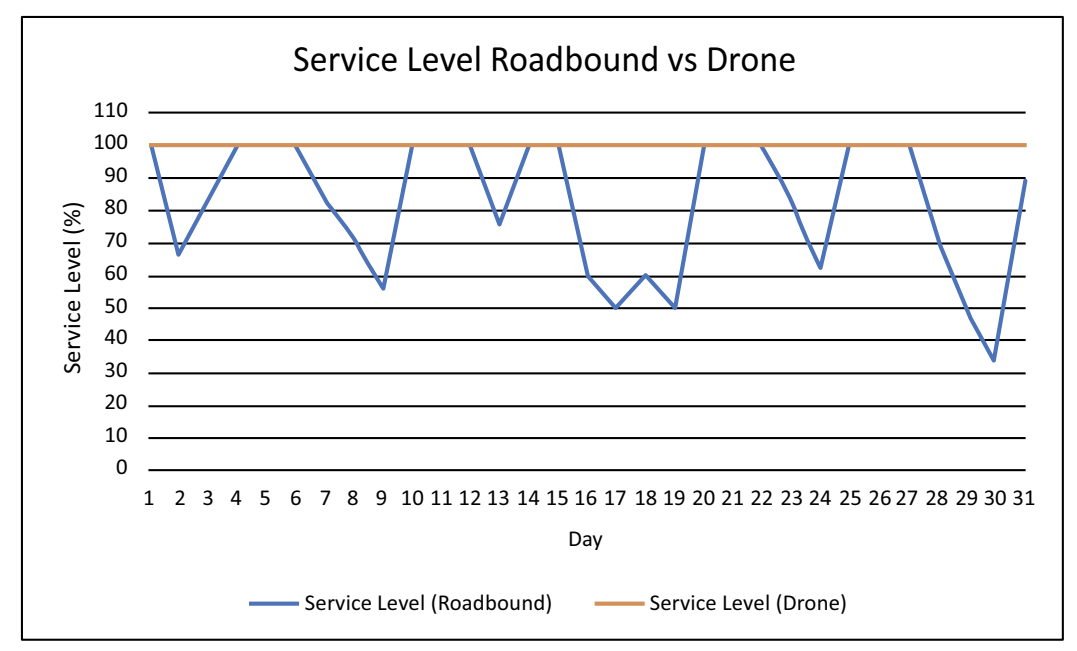

Fig. (8). Service level for all three hospitals combined for each run. 
In the simulation model, a simplification was made that only road-bound vehicles transported the donated hearts. In reality, donated hearts can be transported in many ways. The road-bound vehicles in the simulation did not have the ability to pass traffic, as ambulances can do when turning their sirens on. Air-borne vehicles such as helicopters and airplanes have not been included in this model even though they are possible transportation methods if the organ needs to be transported over a long distance. Adding transportation methods such as ambulance, helicopter and airplane would make the correspondence between the model and reference system more accurate. This would lead to more realistic conclusions on whether transportation with drones is a better transportation method than the methods currently used.

For transportation within a hospital, the simplification was made that it required no time. Of course, this is not the case in any hospital. However, this assumption was made since the likelihood that it would take longer than 1.88 hours to transport an organ within a hospital is small but possible. One could expand this simulation by adding transportation within the hospital if transportation time within hospitals is an issue; however, this was not investigated in this project. This addition would, in that case, increase the accuracy of the simulation.

Each time the simulation runs, new demand will be created. This means that patients who did not receive a heart in that particular run of the simulation are not passed on to the demand in the next run of the simulation. Therefore, the waiting list does not increase even when the demand is not met, and the service level is less than $100 \%$. This is, of course, not the case in reality. To make a more accurate depiction of how the waiting list is affected, the simulation would need to be expanded with a function that continuously adds people to the demand who have not yet received a transplant. In addition, some patients waiting for hearts may die while waiting for a new heart. This should also be incorporated in the demand so as to obtain a more accurate depiction of the real situation.

In fact, since the actual number of available hearts in a day is extremely low, the simulation would need to run for a long time to create a comparison between the two transportation methods, and for this reason, a higher supply was chosen in the simulation. Therefore, the values in the simulation do not depict reality. However, the comparison between service levels by a drone or a car will still be a good depiction. Using roadbound vehicles, it is not always possible for the heart to be transported within a time frame for it. Since patients who are on the waiting list for heart transplantation are in end-stage heart failure, having to wait for another heart can have a fatal outcome.

Use of drones as the transportation method for donated hearts is not only beneficial for Mexico City but is also applicable globally, since transplantation still remains the gold standard in end-stage heart failure. Since drones are not currently a widespread transportation method within healthcare, it is likely that it will take some time before healthcare personnel and society see the potential benefits of using drones, especially with advancements in drone technology and the increasing accuracy of a drone's path and control.
Environmental factors such as weather have not been taken into consideration in the simulation. The environment can slow down the drone or, in a worst-case scenario, can cause total failure. Therefore, there might be additional risks with transporting organs via drones. Further investigation of such effects on different performance indicators, such as cost, patient safety, work environment, etc., needs to be carried out to clearly demonstrate the potential benefits of implementing organ delivery drones in the healthcare system.

\section{CONCLUSION}

Organs for transplantation always have to be transported between the donor and the recipient quickly and professionally. It is common for organs to be transported between hospitals within a city, within a country and even between countries. If the distance and time between the donor and recipient are long, the organs can become unusable. The longer the organ tissue is without blood circulation, the more tissue damage will occur, worsening the chances that the organ will survive and be able to carry out the specific function in the recipient's body. After studying how traffic can affect times between two points, it is clear that road-bound vehicles are not the best way to deliver organs.

It has been shown that, in Mexico City, hearts with a transplantation potential may not be used and may be wasted, as they cannot reach a particular recipient due to the transportation method of road-bound vehicles currently used. Moreover, if drones were to transport the donated organs, there would be more heart transplants made and thus, eventually, the waiting list would decrease at a faster rate. This is because drones are not affected by heavy traffic which can be very severe at peak times in Mexico City.

This project has shown that using drones will increase the safety of transportation, will reduce the heart transplantation waiting list, and eventually, heart transplantation could be made possible in different areas of the city at rush hour times. However, as this simulation did not consider other important factors such as a cost-benefit analysis or a risk analysis, further investigation must be made to conclude how effective the delivery of organs via drone could be in reality. Thus, despite proving that drones have the potential to reach more recipients than road-bound vehicles at a particular time of day, there is much further work to be performed and substantial gaps to be covered in this field, to see whether it is efficient, feasible and reliable to transport hearts by a drone in Mexico City.

\section{ETHICS APPROVAL AND CONSENT TO PARTI- CIPATE}

Not applicable.

\section{HUMAN AND ANIMAL RIGHTS}

No animals/humans were used for studies that are the basis of this research.

\section{CONSENT FOR PUBLICATION}

Not applicable. 


\section{AVAILABILITY OF DATA AND MATERIALS}

The data in this work was extracted from Google Maps namely the website link: https://www.google.se /maps/preview.

\section{FUNDING}

None.

\section{CONFLICT OF INTEREST}

The authors declare no conflict of interest, financial or otherwise.

\section{ACKNOWLEDGEMENTS}

Declared none.

\section{REFERENCES}

[1] M.J. Wilhelm, "Long-term outcome following heart transplantation: Current perspective", J. Thorac. Dis., vol. 7, no. 3, pp. 549-551, 2015. [PMID: 25922738]

[2] J. Treleaven, A. Gennery, J. Marsh, D. Norfolk, L. Page, A. Parker, F. Saran, J. Thurston, and D. Webb, "Guidelines on the use of irradiated blood components prepared by the British Committee for Standards in Haematology blood transfusion task force", Br. J. Haematol., vol. 152, no. 1, pp. 35-51, 2011.

[http://dx.doi.org/10.1111/j.1365-2141.2010.08444.x] [PMID: 21083660]

[3] N.R. Banner, H.L. Thomas, E. Curnow, J.C. Hussey, C.A. Rogers, and R.S. Bonser, "The importance of cold and warm cardiac ischemia for survival after heart transplantation", Transplantation, vol. 86, no. 4, pp. $542-547,2008$.

[http://dx.doi.org/10.1097/TP.0b013e31818149b9] [PMID: 18724223]

[4] U. Aydin, P. Yazici, C. Kazimi, A. Bozoklar, M. Sozbilen, M. Zeytunlu, and M. Kilic, "Simultaneous air transportation of the harvested heart and visceral organs for transplantation", Transplant. Proc., vol. 40, no. 1, pp. 44-46, 2008.

[http://dx.doi.org/10.1016/j.transproceed.2007.12.002] [PMID: 18261543]

[5] J. Wapner, , "Organ droners", Newsweek Global, vol. 166, no. 5, pp. 50-52, 2016.

[6] L. Mantecchini, F. Paganelli, V. Morabito, A. Ricci, D. Peritore, S. Trapani, A. Montemurro, A. Rizzo, E. Del Sordo, A. Gaeta, L. Rizzato, and A. Nanni Costa, "Transportation of organs by air: Safety, quality, and sustainability criteria", Transplant. Proc., vol. 48, no. 2, pp. 304-308, 2016

[http://dx.doi.org/10.1016/j.transproceed.2015.12.050] [PMID: 27109941]

[7] M. Kutzbach, "Megacities and megatraffic", ACCESS Mag., vol. 1, no. 37, pp. 30-35, 2010.

[8] R. Genç, "The logistics management and coordination in procurement phase of organ transplantation", Tohoku J. Exp. Med., vol. 216, no. 4, pp. 287-296, 2008 .

[http://dx.doi.org/10.1620/tjem.216.287] [PMID: 19060443]

[9] M. Balasingam, "Drones in medicine-The rise of the machines", Int. J. Clin. Pract., vol. 71, no. 9, 2017.e12989

[http://dx.doi.org/10.1111/ijcp.12989] [PMID: 28851081]

[10] A. Claesson, A. Bäckman, M. Ringh, L. Svensson, P. Nordberg, T. Djärv, and J. Hollenberg, "Time to delivery of an automated external defibrillator using a drone for simulated out-of-hospital cardiac arrests vs emergency medical services", JAMA, vol. 317, no. 22, pp. 2332-2334, 2017.

[http://dx.doi.org/10.1001/jama.2017.3957] [PMID: 28609525]

[11] A.G. Contreras, "Organ transplantation in Mexico", Transplantation, vol. 100, no. 10, pp. 2011-2012, 2016.

[http://dx.doi.org/10.1097/TP.0000000000001404] [PMID: 27495757]

[12] J. Mckiernan-Gonzáles, "American science, american medicine, american latinos", American Latinos and the Making of the United States, American Latino Theme Study, Science and Medicine. National Park Service, pp. 231-248,

[13] G.R. Caballero, "The demographic transition in Mexico and its implications for the ABC Medical Center", Anales Médicos de la Asociación Médica del Centro Médico ABC, vol. 59, no. 3, pp. 193-196, 2014.

[14] A. Ríos, L. Martínez-Alarcón, M.A. Ayala-García, M.J. Sebastián, A. Abdo-Cuza, A. López-Navas, A. López-López, E.J. Ramírez, G. Muñoz, A. Camacho, J. Suárez-López, R. Castellanos, J.S. Rodríguez, M.A. Martínez, A. Nieto, G. Ramis, P. Ramírez, and P. Parrilla, "Level of acceptance of a clinical solid organ xenotransplantation program among personnel in organ transplant-related services in Spanish, Mexican, and Cuban hospital centers", Transplant. Proc., vol. 42, no. 1, pp. 222-227, 2010.

[http://dx.doi.org/10.1016/j.transproceed.2009.11.007] [PMID: 20172316]

[15] M. Crowley-Matoka, and M. Lock, "Organ transplantation in a globalised world", Mortality, vol. 11, no. 2, pp. 166-181, 2006. [http://dx.doi.org/10.1080/13576270600615310]

[16] K. Miyamoto, S.R. Acharya, M.A. Aziz, J.M. Cusset, T.F. Fwa, H Gerçek, and S. Lee, Transport - environment issues and countermeasures in various metropolises.Urban Transport and the Environment: An International Perspective., Elsevier Science Ltd, 2004, pp. 253-402.

[http://dx.doi.org/10.1108/9780080470290-005]

[17] A. Downs, Still Stuck in Traffic: Coping with Peak-Hour Traffic Congestion., Brookings Institution Press, 2005.

[18] K. Lietz, and L.W. Miller, "Improved survival of patients with endstage heart failure listed for heart transplantation: Analysis of organ procurement and transplantation network/U.S. United Network of Organ Sharing data, 1990 to 2005", J. Am. Coll. Cardiol., vol. 50, no. 13, pp. 1282-1290, 2007.

[http://dx.doi.org/10.1016/j.jacc.2007.04.099] [PMID: 17888847]

[19] P. Widimský, T. Budesínský, D. Vorác, L. Groch, M. Zelízko, M. Aschermann, M. Branny, J. St'ásek, and P. Formánek, 'PRAGUE' Study Group Investigators, "Long distance transport for primary angioplasty $v s$ immediate thrombolysis in acute myocardial infarction. Final results of the randomized national multicentre trial-PRAGUE-2", Eur. Heart J., vol. 24, no. 1, pp. 94-104, 2003. [http://dx.doi.org/10.1016/S0195-668X(02)00468-2] [PMID: 1255 9941]

[20] D.D. Kosambi, The estimation of map distances from recombination values., Springer: New Delhi, 2016, pp. 125-130. [http://dx.doi.org/10.1007/978-81-322-3676-4_16]

[21] Free Map Tools, "How far is it between", Free Map Tools. https:// www.freemaptools.com/how-far-is-it-between.htm

[22] M. Morgan, N. Mays, and W.W. Holland, "Can hospital use be a measure of need for health care?", J. Epidemiol. Community Health, vol. 41 , no. 4 , pp. 269-274, 1987. [http://dx.doi.org/10.1136/jech.41.4.269] [PMID: 3332027]

[23] A. Puri, K.P. Valavanis, and M. Kontitsis, "Statistical profile generation for traffic monitoring using real-time UAV based video data", $M C C A$, IEEE, 2007 pp. 1-6 [http://dx.doi.org/10.1109/MED.2007.4433658]

(C) 2019 Al-Ayyad et al.

This is an open access article distributed under the terms of the Creative Commons Attribution 4.0 International Public License (CC-BY 4.0), a copy of which is available at: https://creativecommons.org/licenses/by/4.0/legalcode. This license permits unrestricted use, distribution, and reproduction in any medium, provided the original author and source are credited. 